\title{
DOUBLING ZETA INTEGRALS AND LOCAL FACTORS FOR METAPLECTIC GROUPS
}

\author{
WEE TECK GAN
}

\section{In memory of Professor Hiroshi Saito}

\begin{abstract}
We develop the theory of the doubling zeta integral of PiatetskiShapiro and Rallis for metaplectic groups $\mathrm{Mp}_{2 n}$, and we use it to give precise definitions of the local $\gamma$-factors, $L$-factors, and $\epsilon$-factors for irreducible representations of $\mathrm{Mp}_{2 n} \times \mathrm{GL}_{1}$, following the footsteps of Lapid and Rallis.
\end{abstract}

\section{$\S 1$. Introduction}

Some twenty-five years ago, Piatetski-Shapiro and Rallis [GPSR, part A] discovered a Rankin-Selberg integral representation for the standard L-function for $G \times \mathrm{GL}_{1}$, where $G$ is a classical group. This Rankin-Selberg integral is called the doubling zeta integral. The associated local zeta integral can be used, à la Tate, to define the local $\gamma$-factor $\gamma(s, \pi \times \chi, \psi)$ for an irreducible representation $\pi \otimes \chi$ of $G \times \mathrm{GL}_{1}$. This was sketched in [PSR], but the definitive treatment only appeared in the recent work of Lapid and Rallis $[\mathrm{LR}]$, which treated the case of symplectic, orthogonal, and unitary groups. There are, however, two classes of classical groups to which the theory of doubling zeta integrals applies but which were not treated in [LR]. The first of these is the class of metaplectic groups $\mathrm{Mp}_{2 n}$, which are the unique 2-fold covers of the symplectic groups $\mathrm{Sp}_{2 n}$. The other is the class of quaternion unitary groups, which are inner forms of symplectic and orthogonal groups (this case has in fact been treated in a recent preprint of Yamana [Y]).

The purpose of this paper is to treat the theory of the doubling zeta integral for the metaplectic group $\mathrm{Mp}_{2 n}$, following the footsteps of Lapid and Rallis [LR]. In particular, given an irreducible genuine representation

Received March 25, 2011. Revised January 6, 2012. Accepted May 2, 2012.

2010 Mathematics Subject Classification. Primary 11F70, 22 E50.

The author's work was partially supported by National Science Foundation grant DMS0801071. 
$\sigma \otimes \chi$ of $\mathrm{Mp}_{2 n} \times \mathrm{GL}_{1}$, we define the local $\gamma$-factor $\gamma(s, \sigma \times \chi, \psi)$, the local $L$-factor $L(s, \sigma, \chi, \psi)$, and the $\epsilon$-factor $\epsilon(s, \sigma \times \chi, \psi)$. Further, we verify that the local $\gamma$-factors satisfy the analogue of the "Ten Commandments" in [LR, Theorem 4], which characterize them uniquely. This theory of local factors is used crucially in the paper [GS], where we study the genuine representation theory of $\mathrm{Mp}_{2 n}$.

We highlight a couple of key differences between the metaplectic case and the linear case.

- In the metaplectic case, the local $L$-factor $L(s, \sigma \times \chi, \psi)$ depends on an additive character $\psi$ of the local field in question, whereas in the linear case, it does not. This reflects the fact that, in the local Langlands correspondence for $\mathrm{Mp}_{2 n}$ (as developed in [GS]), the L-parameter of a representation may change when one changes $\psi$, whereas in the linear case, the L-parameter does not change; only the internal parameterization within an L-packet changes.

- In the symplectic or orthogonal case, the local factors do not change when one replaces the representation of $G$ by its contragredient, whereas in the metaplectic case, they do. More precisely, one has

$$
\gamma\left(s, \sigma^{\vee} \times \chi, \psi\right)=\gamma\left(s, \sigma \times \chi, \psi^{-1}\right)=\gamma\left(s, \sigma \times \chi_{-1} \chi, \psi\right) .
$$

This reflects the fact that, in the symplectic or orthogonal case, the process of taking the contragredient preserves the L-packets, whereas in the metaplectic case, it does not.

We should mention that an important prior work regarding the doubling zeta integral of $\mathrm{Mp}_{2 n}$ is the unpublished preprint by Sweet [Sw]. There, he computed the normalizing factor for an intertwining operator on a degenerate principal series representation. We have benefitted greatly from the results in that work (see Proposition 4.1 below), though at the end of the day, it is possible to establish the results of this paper without resort to $[\mathrm{Sw}]$. Another recent work concerning the doubling zeta integral of $\mathrm{Mp}_{2 n}$ is that of Zorn [Z], though it concerns the evaluation of the zeta integrals on specific test vectors. Finally, an alternative definition of the local $\gamma$-factors for generic representations of $\mathrm{Mp}_{2 n}$ is given in the dissertation of Szpruch $[\mathrm{Sz}]$ via the Langlands-Shahidi method.

\section{$\S 2$. Metaplectic groups}

Let $k$ be a local field of characteristic zero. 


\subsection{Symplectic group}

Let $W$ be a $2 n$-dimensional vector space over $k$ equipped with a nondegenerate skew-symmetric form $\langle-,-\rangle_{W}$, and let $\operatorname{Sp}(W)$ be the associated symplectic group. We may fix a Witt basis of $W$, consisting of vectors

$$
e_{1}, \ldots, e_{n}, e_{n}^{*}, \ldots, e_{1}^{*}
$$

satisfying

$$
\left\langle e_{i}, e_{j}\right\rangle_{W}=\left\langle e_{i}^{*}, e_{j}^{*}\right\rangle_{W}=0 \quad \text { and } \quad\left\langle e_{i}, e_{j}^{*}\right\rangle_{W}=\delta_{i j} .
$$

For any $1 \leq k \leq n$, let

$$
X_{k}=\operatorname{Span}\left(e_{1}, \ldots, e_{k}\right) \quad \text { and } \quad X_{k}^{*}=\operatorname{Span}\left(e_{1}^{*}, \ldots, e_{k}^{*}\right),
$$

so that $W=X_{n} \oplus X_{n}^{*}$. We also set

$$
W_{n-k}=\operatorname{Span}\left(e_{k+1}, \ldots, e_{n}, e_{n}^{*}, \ldots, e_{k+1}^{*}\right)
$$

so that

$$
W=X_{k} \oplus W_{n-k} \oplus X_{k}^{*} .
$$

\subsection{Parabolic subgroups}

We now describe the parabolic subgroups of $\operatorname{Sp}(W)$ up to conjugacy. Consider the flag of isotropic subspaces

$$
X_{k_{1}} \subset X_{k_{1}+k_{2}} \subset \cdots \subset X_{k_{1}+\cdots+k_{r}} \subset W .
$$

The stabilizer of such a flag is a parabolic subgroup $P$ whose Levi factor $M$ is given by

$$
M \cong \mathrm{GL}\left(k_{1}\right) \times \cdots \times \mathrm{GL}\left(k_{r}\right) \times \mathrm{Sp}\left(W_{n-k_{1}-\cdots-k_{r}}\right),
$$

where $\operatorname{GL}\left(k_{i}\right)$ is the group of invertible linear maps on $\operatorname{Span}\left(e_{k_{i-1}+1}, \ldots\right.$, $\left.e_{k_{i-1}+k_{i}}\right)$. In particular, the maximal parabolic subgroups of $\operatorname{Sp}(W)$ are simply the stabilizers $P\left(X_{k}\right)$ of the isotropic spaces $X_{k}(1 \leq k \leq n)$. For a given $k$, a Levi subgroup of $P\left(X_{k}\right)$ is given by

$$
M\left(X_{k}\right)=\mathrm{GL}\left(X_{k}\right) \times \operatorname{Sp}\left(W_{n-k}\right),
$$

and its unipotent radical $N\left(X_{k}\right)$ sits in a short exact sequence

$$
1 \longrightarrow Z\left(X_{k}\right) \longrightarrow N\left(X_{k}\right) \longrightarrow \operatorname{Hom}\left(W_{n-k}, X_{k}\right) \longrightarrow 1,
$$

where $Z\left(X_{k}\right) \cong \operatorname{Sym}^{2} X_{k}$ is isomorphic to the space of symmetric bilinear form on $Y_{k}$. When $k=n, N\left(X_{k}\right)=Z\left(X_{k}\right)$ is abelian, and $P\left(X_{n}\right)$ is called the Siegel parabolic subgroup. 


\subsection{Metaplectic group}

The group $\operatorname{Sp}(W)$ has a unique 2 -fold cover $\operatorname{Mp}(W)$. As a set, we may write

$$
\operatorname{Mp}(W)=\operatorname{Sp}(W) \times\{ \pm 1\},
$$

with group law given by

$$
\left(g_{1}, \epsilon_{1}\right) \cdot\left(g_{2}, \epsilon_{2}\right)=\left(g_{1} g_{2}, \epsilon_{1} \epsilon_{2} \cdot c\left(g_{1}, g_{2}\right)\right)
$$

for some 2-cocycle $c$ on $\operatorname{Sp}(W)$ valued in $\{ \pm 1\}$, as described by Rao $[\mathrm{R}]$. In the following, we will typically write $\mu_{2}$ to denote the subgroup $\{ \pm 1\}$ of $\operatorname{Mp}(W)$; it is the preimage of the identity element of $\operatorname{Sp}(W)$. Without describing $c$ explicitly, let us describe the restriction of this double cover over a maximal parabolic subgroup $P\left(X_{k}\right)$ of $\operatorname{Sp}(W)$.

The covering splits uniquely over the unipotent radical $N\left(X_{k}\right)$ of $P\left(X_{k}\right)$. Thus, we may regard $N\left(X_{k}\right)$ canonically as a subgroup of $\operatorname{Mp}(W)$, and one has a Levi decomposition

$$
\widetilde{P}\left(X_{k}\right)=\widetilde{M}\left(X_{k}\right) \cdot N\left(X_{k}\right) .
$$

We need to describe the covering over $M\left(X_{k}\right) \cong \mathrm{GL}\left(X_{k}\right) \times \operatorname{Sp}\left(W_{n-k}\right)$.

Not surprisingly, the restriction of the covering to $\operatorname{Sp}\left(W_{n-k}\right)$ is nothing but the unique 2-fold cover $\operatorname{Mp}\left(W_{n-k}\right)$ of $\operatorname{Sp}\left(W_{n-k}\right)$. The covering over $\mathrm{GL}\left(X_{k}\right)$ can be described as follows. Consider the set

$$
\mathrm{GL}\left(X_{k}\right) \times\{ \pm 1\}
$$

with multiplication law

$$
\left(g_{1}, \epsilon_{1}\right) \cdot\left(g_{2}, \epsilon_{2}\right)=\left(g_{1} g_{2}, \epsilon_{1} \epsilon_{2} \cdot\left(\operatorname{det} g_{1}, \operatorname{det} g_{2}\right)\right),
$$

where $\left(\operatorname{det} g_{1}, \operatorname{det} g_{2}\right)$ denotes the Hilbert symbol. Then $\widetilde{\mathrm{GL}}\left(X_{k}\right)$ is precisely this double cover of $\mathrm{GL}\left(X_{k}\right)$.

Hence, we have

$$
\widetilde{M}\left(X_{k}\right)=\left(\widetilde{\mathrm{GL}}\left(X_{k}\right) \times \operatorname{Mp}\left(W_{n-k}\right)\right) / \Delta \mu_{2}=: \widetilde{\mathrm{GL}}\left(X_{k}\right) \times_{\mu_{2}} \operatorname{Mp}\left(W_{n-k}\right),
$$

where $\Delta \mu_{2}$ is the diagonally embedded $\mu_{2}$. More generally, for any parabolic subgroup $P$, one has the Levi decomposition

$$
\widetilde{P}=\widetilde{M} \cdot N
$$

with

$$
\widetilde{M} \cong \widetilde{\mathrm{GL}}\left(k_{1}\right) \times_{\mu_{2}} \cdots \times_{\mu_{2}} \widetilde{\mathrm{GL}}\left(k_{r}\right) \times_{\mu_{2}} \operatorname{Mp}\left(W_{n-k_{1}-\cdots-k_{r}}\right) .
$$




\subsection{Representations of $\widetilde{\mathrm{GL}}\left(X_{k}\right)$}

The (genuine) representation theory of $\widetilde{\mathrm{GL}}\left(X_{k}\right)$ can be easily related to the representation theory of $\operatorname{GL}\left(X_{k}\right)$. Indeed, the determinant map

$$
\operatorname{det}: \mathrm{GL}\left(X_{k}\right) \longrightarrow \mathrm{GL}(1)
$$

has a natural lifting

$$
\widetilde{\operatorname{det}}: \widetilde{\mathrm{GL}}\left(X_{k}\right) \longrightarrow \widetilde{\mathrm{GL}}(1)
$$

given by

$$
\widetilde{\operatorname{det}}(g, \epsilon)=(\operatorname{det} g, \epsilon) .
$$

On the other hand, if we fix an additive character $\psi$ of $k$, then there is a natural genuine character of $\widetilde{\mathrm{GL}}(1)$ defined by

$$
(a, \epsilon) \mapsto \epsilon \cdot \gamma(a, \psi)^{-1},
$$

with

$$
\gamma(a, \psi)=\gamma\left(\psi_{a}\right) / \gamma(\psi)
$$

and the Weil index $\gamma(\psi)$ is an eighth root of unity associated to $\psi$ by Weil. Composing this genuine character by $\widetilde{\text { det }}$ gives a genuine character $\chi_{\psi}$ of $\widetilde{\mathrm{GL}}\left(X_{k}\right)$, which satisfies

$$
\chi_{\psi}(g, \epsilon)^{2}=(\operatorname{det} g,-1) .
$$

While it is more accurate to denote this genuine character by $\chi_{\psi} \circ \widetilde{\mathrm{det}}$, we will abuse notation in writing $\chi_{\psi}$ for the sake of simplicity, unless there is a possible confusion. Using the genuine character $\chi_{\psi}$, one obtains a bijection between $\operatorname{Irr}\left(\mathrm{GL}\left(X_{k}\right)\right)$ and the set $\operatorname{Irr}\left(\widetilde{\mathrm{GL}}\left(X_{k}\right)\right)$ of genuine irreducible representations of $\widetilde{\mathrm{GL}}\left(X_{k}\right)$ via

$$
\tau \mapsto \tilde{\tau}_{\psi}=\tau \otimes \chi_{\psi}
$$

We stress that this bijection depends on the choice of the additive character $\psi$. More precisely, if $\psi_{a}$ is the additive character defined by $\psi_{a}(x)=\psi(a x)$ (with $a \in k^{\times}$), then

$$
\chi_{\psi_{a}}=\chi_{\psi} \cdot \chi_{a}
$$

where $\chi_{a}$ is the quadratic character of $k^{\times}$associated to $a$, so that

$$
\tilde{\tau}_{\psi_{a}}=\tilde{\tau}_{\psi} \otimes\left(\chi_{a} \circ \text { det }\right)
$$




\subsection{Parabolic induction}

After the above discussion, one sees that given an irreducible representation $\tau$ of $\operatorname{GL}\left(X_{k}\right)$ and an irreducible representation $\pi$ of $\operatorname{Mp}\left(W_{n-k}\right)$, one has an irreducible representation $\tilde{\tau}_{\psi} \otimes \pi$ of $\widetilde{M}\left(X_{k}\right)$. Thus, one may consider the parabolically induced representation

$$
I_{P\left(X_{k}\right), \psi}(\tau, \pi)=\operatorname{Ind}_{\widetilde{P}\left(X_{k}\right)}^{\mathrm{Mp}(W)} \tilde{\tau}_{\psi} \otimes \pi \quad \text { (normalized induction). }
$$

More generally, for any parabolic subgroup $P=M \cdot N$ and irreducible representation $\tau_{i}$ of $\mathrm{GL}\left(k_{i}\right)$ and $\pi$ of $\operatorname{Mp}\left(W_{n-k_{1}-\cdots-k_{r}}\right)$, one has the induced representation

$$
I_{P, \psi}\left(\tau_{1}, \ldots, \tau_{r}, \pi\right)
$$

A particular case of this is when $P=B$ is the Borel subgroup, so that each $k_{i}=1$. In that case, given characters $\chi_{1}, \ldots, \chi_{n}$, one has the principal series representations

$$
I_{B, \psi}\left(\chi_{1}, \ldots, \chi_{n}\right)
$$

If the $\chi_{i}$ are unramified, we will call such a representation an unramified principal series representation; note that this notion of unramified representations depends on the choice of $\psi$. Indeed, one has

$$
I_{B, \psi_{a}}\left(\chi_{1}, \ldots, \chi_{n}\right)=I_{B, \psi}\left(\chi_{1} \chi_{a}, \ldots, \chi_{n} \chi_{a}\right)
$$

\subsection{Central signs}

We could restrict the genuine character $\chi_{\psi}$ of $\tilde{\mathrm{GL}}\left(X_{n}\right)$ to the center $\widetilde{Z}$ (the preimage in $\operatorname{Mp}(W)$ of the center $Z$ of $\operatorname{Sp}(W)$ ) of $\operatorname{Mp}(W)$. We denote this character of $\widetilde{Z}$ by $\chi_{\psi}$ as well. This character allows one to define a central sign for irreducible genuine representations $\sigma$ of $\mathrm{Mp}(W)$ as follows.

For an irreducible genuine representation $\sigma$ of $\operatorname{Mp}(W)$, one may consider its central character $\omega_{\sigma}$, which is a genuine character of $\tilde{Z}$. We define the central $\operatorname{sign} z_{\psi}(\sigma)$ of $\sigma$ by

$$
z_{\psi}(\sigma)=\omega_{\sigma}(-1) / \chi_{\psi}(\widetilde{\operatorname{det}}(-1)) \in\{ \pm 1\}
$$

where we note that the quotient above is independent of the choice of the preimage in $\tilde{Z}$ of $-1 \in Z$. 


\section{$\S 3$. Doubling zeta integrals}

We now recall the basic definition of the doubling zeta integral. Set $\mathbb{W}=$ $W+(-W)$, so that there is a natural map

$$
\operatorname{Mp}(W) \times_{\mu_{2}} \operatorname{Mp}(W) \hookrightarrow \operatorname{Mp}(\mathbb{W}) .
$$

Consider the maximal isotropic subspaces

$$
W^{\Delta}=\{(w, w): w \in W\} \quad \text { and } \quad W^{\nabla}=\{(w,-w): w \in W\},
$$

so that $\mathbb{W}=W^{\Delta}+W^{\nabla}$. The maximal parabolic subgroup of $\mathrm{Sp}(\mathbb{W})$ stabilizing $W^{\Delta}$ is

$$
P\left(W^{\Delta}\right)=\mathrm{GL}\left(W^{\Delta}\right) \cdot N\left(W^{\Delta}\right) .
$$

Passing to covering groups, one has

$$
\tilde{P}\left(W^{\Delta}\right)=\tilde{G L}\left(W^{\Delta}\right) \cdot N\left(W^{\Delta}\right) \subset \mathrm{Mp}(\mathbb{W}) .
$$

Henceforth, fix a nontrivial additive character $\psi$ of $F$. Then $\psi$ determines a genuine character $\chi_{\psi}$ of $\tilde{G L}\left(W^{\Delta}\right)$ as explained in Section 2.6. For any character $\chi$ of $F^{\times}$, one can define the degenerate principal series representation

$$
I_{\tilde{P}\left(W^{\Delta}\right), \psi}(s, \chi):=\operatorname{Ind}_{\tilde{P}\left(W^{\Delta}\right)}^{\mathrm{Mp}(\mathbb{W})} \chi_{\psi} \cdot(\chi \circ \operatorname{det})|\operatorname{det}|^{s} \quad \text { (normalized induction). }
$$

Now let $\sigma$ be an irreducible genuine representation of $\mathrm{Mp}(W)$ with contragredient $\sigma^{\vee}$. One has a natural $\operatorname{Mp}(W)$-invariant pairing $\langle-,-\rangle$ on $\sigma^{\vee} \otimes \sigma$. For $v \in \sigma, v^{\vee} \in \sigma^{\vee}$, and a holomorphic section $f_{s} \in I_{\tilde{P}\left(W^{\Delta}\right), \psi}(s, \chi)$, the doubling zeta integral is defined for $\operatorname{Re}(s) \gg 0$ by

$$
Z_{W, \psi}(s, \chi)\left(f, v^{\vee}, v\right)=\int_{\mathrm{Mp}(W)} f_{s}(g, 1) \cdot\left\langle g v^{\vee}, v\right\rangle d g
$$

for a fixed Haar measure $d g$ on $\operatorname{Mp}(W)$. Note here that we have used the matrix coefficient $\left\langle g v^{\vee}, v\right\rangle$ of $\sigma^{\vee}$ rather than the matrix coefficient $\left\langle g v, v^{\vee}\right\rangle$ of $\sigma$. Thus, $Z_{W, \psi}(s, \chi)$ defines an $\operatorname{Mp}(W) \times \mathrm{Mp}(W)$-invariant linear form

$$
Z_{W, \psi}(s, \chi): I_{\tilde{P}\left(W^{\Delta}\right), \psi}(s, \chi) \otimes \sigma^{\vee} \otimes \sigma \longrightarrow \mathbb{C} .
$$

The analytic properties of this zeta integral, as a function of $s$, are summarized as follows. 
THEOREM 3.1. One has the following.

(i) There exists a constant $c$ such that whenever $\operatorname{Re}(s)>c$, the integral $Z_{W, \psi}(s, \chi)\left(f, v^{\vee}, v\right)$ converges for all data $f, v$, and $v^{\vee}$. If $\pi$ is tempered, then we may take $c=-1 / 2$.

(ii) If $f_{s}$ is a holomorphic section of $I_{\tilde{P}\left(W^{\Delta}\right), \psi}(s, \chi)$, then the function $Z_{W, \psi}(s, \chi)\left(f, v^{\vee}, v\right)$ admits meromorphic continuation to $\mathbb{C}$. When $k$ is $p$-adic, $Z_{W, \psi}(s, \chi)\left(f, v, v^{\vee}\right)$ is a rational function in $q^{-s}$ (where $q$ is the cardinality of the residue field of $k$ ).

(iii) For each $s_{0}$, there exist data $f, v$, and $v^{\vee}$ such that $Z_{W, \psi}(s, \chi)\left(f, v^{\vee}, v\right)$ is finite and nonzero.

(iv) There is a nonnegative integer $k$ (depending on $s_{0}$ ) such that ( $s-$ $\left.s_{0}\right)^{k} \cdot Z_{W, \psi}(s, \chi)\left(f, v^{\vee}, v\right)$ is holomorphic at $s=s_{0}$ for all choices of data $\left(f, v, v^{\vee}\right)$ and is nonzero there for some choices of data.

(v) Let $Z_{W, \psi}^{*}\left(s_{0}, \chi\right)$ denote the leading term in the Laurent expansion at $s=s_{0}$ of $Z_{W, \psi}(s, \chi)$ as a linear form, so that

$$
Z_{W, \psi}^{*}\left(s_{0}, \chi\right)\left(f, v^{\vee}, v\right)=\left.\left(\left(s-s_{0}\right)^{k} \cdot Z_{W, \psi}(s, \chi)\left(f, v^{\vee}, v\right)\right)\right|_{s=s_{0}} .
$$

Then $Z_{W, \psi}^{*}\left(s_{0}, \chi\right)$ is a nonzero element of

$$
\operatorname{Hom}_{\mathrm{Mp}(W) \times \mathrm{Mp}(W)}\left(I_{\tilde{P}\left(W^{\Delta}\right), \psi}\left(s_{0}, \chi\right) \otimes \sigma^{\vee} \otimes \sigma, \mathbb{C}\right) .
$$

\section{$\S 4$. Normalization of intertwining operator}

We continue with the notation of the previous section. One has an intertwining operator

$$
M(\chi, s): I_{\tilde{P}\left(W^{\Delta}\right), \psi}(s, \chi) \longrightarrow I_{\tilde{P}\left(W^{\Delta}\right), \psi}\left(-s, \chi^{-1}\right)
$$

defined by

$$
M(\chi, s)(f)(g)=\int_{N\left(W^{\Delta}\right)} f(w n g) d n
$$

where

$$
w=\left(1_{W},-1_{W}\right) \in \operatorname{Mp}(W) \times_{\mu_{2}} \operatorname{Mp}(W) .
$$

This integral converges for $\operatorname{Re}(s)$ sufficiently large and has a meromorphic continuation to all of $\mathbb{C}$. Note that this intertwining operator depends on the choice of the Haar measure $d n$ on $N\left(W^{\Delta}\right)$. In [Sw] and [Z], a normalization of $M(\chi, s)$ is given as in [LR], and we recall its definition briefly. 
We fix a nondegenerate character on the abelian group $N\left(W^{\nabla}\right) \cong$ $\mathrm{Sym}^{2} W^{\nabla}$ as follows. To every element

$$
A \in N\left(W^{\Delta}\right) \subset \operatorname{Hom}\left(W^{\nabla}, W^{\Delta}\right),
$$

one obtains a character $\psi_{A}$ of $N\left(W^{\nabla}\right)$ defined by

$$
\psi_{A}(X)=\operatorname{Tr}_{W} \nabla(X \circ A) .
$$

If one identifies $W^{\nabla} \cong W$ via $(w,-w) \mapsto 2 w$ and $W^{\Delta} \cong W$ via $(w, w) \mapsto w$ (following $[\mathrm{LR}]$ ), then $A: W \longrightarrow W$, and so one may consider $\operatorname{det} A$.

Henceforth, we assume that $A$ corresponds to the split nondegenerate quadratic form on the even-dimensional space $W^{\Delta}$, in which case we may assume that $\operatorname{det} A \in(-1)^{n} \cdot F^{\times 2}$.

Now one can show (see [Z, Appendix]) that, for all $s$,

$$
\operatorname{dim} \operatorname{Hom}_{N\left(W^{\nabla}\right)}\left(I_{\tilde{P}\left(W^{\Delta}\right), \psi}(s, \chi), \psi_{A}^{-1}\right)=1 .
$$

Moreover, a nonzero element of this 1-dimensional space can be given as a generalized Jacquet integral:

$$
\mathcal{W}_{\psi, A}(\chi, s)(f)=\int_{N\left(W^{\nabla}\right)} f(u) \cdot \psi_{A}(u) d u .
$$

This integral converges for $\operatorname{Re}(s)$ sufficiently large and has an analytic continuation to $\mathbb{C}$. Note that $\mathcal{W}_{\psi, A}(\chi, s)$ depends on the choice of the Haar measure $d u$.

By the multiplicity 1 result above, there is a meromorphic function $c_{\psi, A}(\chi$, $s$ ) such that

$$
\mathcal{W}_{\psi, A}\left(\chi^{-1},-s\right) \circ M(\chi, s)=c_{\psi, A}(\chi, s) \cdot \mathcal{W}_{\psi, A}(\chi, s)
$$

The explicit determination of the function $c_{\psi, A}(\chi, s)$ has been given in [Sw] (see also [Z]). We will recall the answer in the unramified case below. In general, the following consequence of Sweet's results is sufficient for our purpose.

Proposition 4.1. We have

$$
c_{\psi, A}\left(s, \chi \chi_{a}\right)=\chi_{a}(-1)^{n} \cdot c_{\psi, A}(s, \chi)
$$


for any quadratic character $\chi_{a}$ (associated to $\left.a \in F^{\times}\right)$,

$$
c_{\psi_{a}, A}(s, \chi)=c_{\psi}(s, \chi) \cdot \chi(a)^{-2 n} \cdot|a|^{-2 n s} \cdot \chi_{a}(-1)^{n},
$$

and

$$
c_{\psi, a \cdot A}(s, \chi)=c_{\psi, A}(s, \chi) \cdot \chi(a)^{-2 n}|a|^{-2 n s} .
$$

Indeed, we give an independent proof of this proposition later in this article.

Now one may normalize the intertwining operator $M(\chi, s)$ by setting

$$
M_{\psi, A}^{*}(\chi, s)=c_{\psi, A}(\chi, s)^{-1} \cdot M(\chi, s)
$$

so that

$$
\mathcal{W}_{\psi, A}\left(\chi^{-1},-s\right) \circ M_{\psi, A}^{*}(\chi, s)=\mathcal{W}_{\psi, A}(\chi, s) .
$$

Then this normalized operator is independent of the choice of the Haar measure $d n$ or $d u$. Moreover, it satisfies

$$
M_{\psi, A}^{*}\left(\chi^{-1},-s\right) \circ M_{\psi, A}^{*}(\chi, s)=1 .
$$

The above proposition implies the following.

Corollary 4.2. As linear maps from $I_{\tilde{P}\left(W^{\Delta}\right), \psi}(s, \chi)$ to $I_{\tilde{P}\left(W^{\Delta}\right), \psi}(-s$, $\left.\chi^{-1}\right)$, one has

$$
M_{\psi, a \cdot A}^{*}(\chi, s)=\chi(a)^{2 n} \cdot|a|^{2 n s} \cdot M_{\psi, A}^{*}(\chi, s) .
$$

\section{$\S 5$. Definition of local factors}

It can be shown that, for generic $s \in \mathbb{C}$,

$$
\operatorname{dim} \operatorname{Hom}_{\mathrm{Mp}(W) \times \operatorname{Mp}(W)}\left(I_{\tilde{P}\left(W^{\Delta}\right), \psi}(s, \chi) \otimes \sigma^{\vee} \otimes \sigma, \mathbb{C}\right)=1 .
$$

Thus, one obtains a functional equation

$$
\begin{aligned}
& Z_{W, \psi}\left(-s, \chi^{-1}\right) \circ\left(M_{\psi, A}^{*}(\chi, s) \otimes 1_{\sigma} \otimes 1_{\sigma^{\vee}}\right) \\
& \quad=z_{\psi}(\sigma) \cdot \chi(\operatorname{det} A) \cdot|\operatorname{det}(A)|^{s} \cdot \gamma_{W}\left(s+\frac{1}{2}, \sigma \times \chi, A, \psi\right) \cdot Z_{W, \psi}(s, \chi)
\end{aligned}
$$

for some meromorphic function $\gamma_{W}(s+1 / 2, \sigma \times \chi, A, \psi)$. It follows from Corollary 4.2 that $\gamma_{W}(s, \sigma \times \chi, A, \psi)$ is independent of the choice of $A$, and so we may denote it by $\gamma_{W}(s, \sigma \times \chi, \psi)$. 
Definition. We have the following.

(i) The function $\gamma_{W}(s, \sigma \times \chi, \psi)$ is called the standard $\gamma$-factor of $\sigma \otimes \chi$.

(ii) If $\sigma$ is tempered, we may write

$$
\gamma_{W}(s, \sigma \times \chi, \psi)=\epsilon(s, \sigma \times \chi, \psi) \cdot \frac{L\left(1-s, \sigma^{\vee} \times \chi^{-1}, \psi\right)}{L(s, \sigma \times \chi, \psi)},
$$

where $\epsilon(s, \sigma \times \chi, \psi)$ is a monomial function of $q^{-s}$ and $L(s, \sigma \times \chi, \psi)^{-1}$ is the numerator of the rational function $\gamma(s, \sigma \times \chi, \psi)$, normalized so that it is a polynomial in $q^{-s}$ with constant term 1 . The function $\epsilon(s, \sigma \times \chi, \psi)$ is called the standard $\epsilon$-factor of $\sigma \otimes \chi$, and $L(s, \sigma \times \chi, \psi)$ is the standard L-factor of $\sigma \otimes \chi$ relative to the choice of $\psi$.

(iii) If $\sigma$ is nontempered, we realize $\sigma$ as a Langlands quotient of a standard module and define $\epsilon(s, \sigma \times \chi, \psi)$ and $L(s, \sigma \times \chi, \psi)$ by multiplicativity (following Shahidi). More precisely, if $\sigma$ is a Langlands quotient of $I_{P, \psi}\left(\tau_{1}, \ldots, \tau_{r}, \pi\right)$, with $\tau_{i}$ essentially tempered representations of $\mathrm{GL}\left(k_{i}\right)$ and $\pi$ a tempered representation of $\operatorname{Mp}\left(W_{n-k_{1}-\cdots-k_{r}}\right)$, then we set

$$
L(s, \sigma \times \chi, \psi)=L(s, \pi \times \chi, \psi) \cdot\left(\prod_{i=1}^{r} L\left(s, \tau_{i} \times \chi\right) \cdot L\left(s, \tau_{i}^{\vee} \times \chi\right)\right),
$$

and likewise for $\epsilon(s, \sigma \times \chi, \psi)$. Here $L\left(s, \tau_{i} \times \chi\right)$ stands for the usual Rankin-Selberg L-function for GL $\left(k_{i}\right) \times \mathrm{GL}(1)$ (which can be defined by various different approaches: Godement-Jacquet, Langlands-Shahidi, or Rankin-Selberg). The results of Section 7 show that with this definition, one still has the same formula relating $\gamma(s, \sigma \times \chi, \psi)$ to the $L$-factor and $\epsilon$-factor as in (ii) above.

It follows from the definition that

$$
\gamma_{W}\left(s, \sigma \times \chi|-|^{t}, \psi\right)=\gamma_{W}(s+t, \sigma \times \chi, \psi) .
$$

The rest of this article is devoted to verifying other less obvious properties of $\gamma_{W}(s, \sigma \times \chi, \psi)$. The first order of business is to understand the unramified case thoroughly; we do this in the next section.

We remark that an alternative (and popular) definition of local $L$-factors is as the greatest common divisor of the local zeta integrals for the space of good sections. Indeed, this was the original approach sketched in [PSR]. This alternative approach has now been developed for all classical groups 
in a recent preprint of Yamana $[\mathrm{Y}]$. While the local L-factor defined via this approach is designed to control the poles of the local zeta integrals, the one defined above following Lapid and Rallis [LR] has the advantage that it has the right properties with respect to the local Langlands correspondence; that is, it agrees with the corresponding L-factor on the Galois side. While both approaches give the same $\gamma$-factors, it is not at all clear that they give the same $L$-factors. Thankfully, the main result of $[Y]$ says that the L-factors supplied by both approaches are in fact equal.

\section{§6. Unramified case}

Assume that the residue characteristic $p$ of $F$ is odd, that all the data involved are unramified, that is, that $\sigma$ and $\chi$ are unramified representations, and that the conductor of $\psi$ is the ring of integers $\mathcal{O}_{F}$ of $F$ and $\operatorname{det} A \in \mathcal{O}_{F}^{\times}$. Then one has

$$
\sigma \subset I_{\tilde{B}, \psi}\left(\chi_{1}, \ldots, \chi_{n}\right)
$$

for some unramified characters $\chi_{i}$ of $F^{\times}$. In this case, one would like to check that

$$
L(s, \sigma \times \chi, \psi)=L^{*}(s, \sigma \times \chi, \psi):=\prod_{i=1}^{n} L\left(s, \chi_{i} \chi\right) \cdot L\left(s, \chi_{i}^{-1} \chi\right) .
$$

However, this is not immediate from the definition. One can show this desired equality by an explicit calculation of the various quantities used in the definition of the $\gamma$-factor. The results are summarized in the following proposition.

Proposition 6.1. Suppose that $p$ is odd and that $\sigma, \chi, \psi$, and $A$ are unramified. Let $f_{0}, v_{0}$, and $v_{0}^{\vee}$ be the spherical vectors in $I_{\tilde{P}\left(W^{\Delta}\right), \psi}(s, \chi), \sigma$, and $\sigma^{\vee}$, respectively.

(i) One has

$$
Z_{W, \psi}(s, \chi)\left(f_{0}, v_{0}^{\vee}, v_{0}\right)=\frac{L^{*}(s, \sigma \times \chi, \psi)}{d(\chi, s)}
$$

where

$$
d(\chi, s)=\prod_{k=1}^{n} L\left(2 s,+2 k, \chi^{2}\right)
$$


(ii) One has

$$
\mathcal{W}_{\psi, A}(\chi, s)\left(f_{0}\right)=\frac{1}{d(\chi, s)}
$$

(iii) One has

$$
M(\chi, s)\left(f_{0}\right)=\prod_{j=1}^{n} \frac{L\left(2 s-2 j+1, \chi^{2}\right)}{L\left(2 s+2 j, \chi^{2}\right)} .
$$

(iv) One has

$$
c_{\psi, A}(\chi, s)=\prod_{j=1}^{n} \gamma\left(2 s-(2 j-1), \chi^{2}, \psi\right)^{-1},
$$

where the $\gamma$-factors on the right-hand side are those of Tate. In particular, one has

$$
M_{\psi, A}^{*}(\chi, s)\left(f_{0}\right)=\frac{d\left(\chi^{-1},-s\right)}{d(\chi, s)} \cdot f_{0} .
$$

Proof. The statement (i) was shown by Li [Li, Proposition 4.6]; (ii) can be shown in the same way as in [LR, Proposition 4]; (iii) was shown by Zorn [Z, Section 4]; and (iv) was shown by Sweet [Sw], although it also follows from (ii) and (iii).

Corollary 6.2. Assume the same hypotheses as the above proposition. Then one has

$$
\gamma_{W}(s, \sigma \times \chi, \psi)=\frac{L^{*}\left(1-s, \sigma \times \chi^{-1}, \psi\right)}{L^{*}(s, \sigma \times \chi, \psi)} .
$$

In particular, one has

$$
L(s, \sigma \times \chi, \psi)=L^{*}(s, \sigma \times \chi, \psi) .
$$

Proof. The first assertion follows from Proposition 6.1 and the definition of $\gamma_{W}(s, \sigma \times \chi, \psi)$, on noting that $z_{\psi}(\sigma)=1$. For the second assertion, suppose that

$$
\sigma \subset I_{\tilde{B}, \psi}\left(\chi_{1}, \ldots, \chi_{n}\right) .
$$

If $\sigma$ is tempered and $\chi$ is unitary, then $\chi_{i}$ is unitary for each $i$, and hence there can be no cancellation of factors in $L^{*}\left(1-s, \sigma \times \chi^{-1}, \psi\right)$ with those of $L^{*}(s, \sigma \times \chi, \psi)$. This implies the desired equality in the tempered case. The nontempered case follows by definition and the fact that every nontempered unramified $\sigma$ is the unique Langlands quotient of an unramified standard module. 


\section{$\S 7$. Doubling zeta integral for $\tilde{\mathrm{GL}}(X)$}

In order to establish the crucial property of multiplicativity of the $\gamma$-factors defined above, it is necessary to consider the doubling zeta integral associated to a representation of a Levi subgroup $M$ of $\operatorname{Mp}(W)$. If $X \subset W$ is an isotropic subspace, with $W=X+W_{0}+X^{*}$, and $P(X)=M(X) \cdot N(X)$ is the associated maximal parabolic, then $M(X) \cong \tilde{\mathrm{GL}}(X) \times{ }_{\mu_{2}} \mathrm{Mp}\left(W_{0}\right)$. Thus, we need to consider the doubling zeta integral associated to a genuine irreducible representation of $\tilde{\mathrm{GL}}(X)$. The nonlinear group $\tilde{\mathrm{GL}}(X)$ and its genuine representations were described in Section 2.

\subsection{Zeta integral for $\tilde{\mathrm{GL}}(X)$}

Let $X$ be a vector space, and set $\mathbb{X}=X \oplus X$. Let

$$
X^{\Delta}=\{(x, x): x \in X\} \subset \mathbb{X},
$$

and let

$$
X^{\nabla}=\{(x,-x): x \in X\}
$$

so that

$$
\mathbb{X}=X^{\Delta} \oplus X^{\nabla}
$$

Let $P\left(X^{\Delta}\right)$ be the parabolic subgroup of $\mathrm{GL}(\mathbb{X})$ stabilizing $X^{\Delta}$. The Levi subgroup of $P\left(X^{\Delta}\right)$ is

$$
M\left(X^{\Delta}\right)=\mathrm{GL}\left(X^{\Delta}\right) \times \mathrm{GL}\left(X^{\nabla}\right)
$$

and its unipotent radical is

$$
N\left(X^{\Delta}\right)=\operatorname{Hom}\left(X^{\nabla}, X^{\Delta}\right) .
$$

For ease of notation, we will simply write det for the rational character $\operatorname{det}_{X^{\Delta}} \times \operatorname{det}_{X \nabla}^{-1}$ of $M\left(X^{\Delta}\right)$. Then one has the degenerate principal series representation

$$
I_{\tilde{P}\left(X^{\Delta}\right), \psi}(\chi, s):=\operatorname{Ind}_{\tilde{P}\left(X^{\Delta}\right)} \chi_{\psi} \cdot(\chi \circ \operatorname{det}) \cdot|\operatorname{det}|^{s}
$$

of $\tilde{\mathrm{GL}}(\mathbb{X})$.

We may now repeat the construction of the doubling zeta integral. Let $\tau$ be an irreducible representation of $\mathrm{GL}(X)$, and set

$$
\tau_{\psi}:=\chi_{\psi} \otimes \tau
$$


so that $\tau_{\psi}$ is a genuine representation of $\tilde{\mathrm{GL}}(X)$. Then one sets

$$
\tilde{Z}_{X, \psi}(s, \chi)\left(\tilde{f}, v^{\vee}, v\right)=\int_{\tilde{\mathrm{GL}}(X)} \tilde{f}_{s}(g, 1) \cdot\left\langle\tau_{\psi}^{\vee}(g) v^{\vee}, v\right\rangle \cdot d g
$$

for $\tilde{f}_{s} \in I_{\tilde{P}\left(X^{\Delta}\right), \psi}(\chi, s), v \in \tau_{\psi}$, and $v^{\vee} \in \tau_{\psi}^{\vee}$. This is the doubling zeta integral for the representation $\tau_{\psi} \otimes \chi$ of $\tilde{\mathrm{GL}}(X) \times \mathrm{GL}_{1}$, and it defines a $\tilde{\mathrm{GL}}(X) \times$ $\tilde{\mathrm{GL}}(X)$-invariant form

$$
\tilde{Z}_{X, \psi}(s, \chi): I_{\tilde{P}\left(X^{\Delta}\right), \psi}(\chi, s) \otimes \tau_{\psi}^{\vee} \otimes \tau_{\psi} \longrightarrow \mathbb{C} .
$$

As before, one has a standard intertwining operator

$$
\tilde{M}(\chi, s): I_{\tilde{P}\left(X^{\Delta}\right), \psi}(\chi, s) \longrightarrow I_{\tilde{P}\left(X^{\Delta}\right), \psi}\left(\chi^{-1},-s\right)
$$

and one may define an normalized intertwining operator $\tilde{M}_{\psi}^{*}(\chi, s)$ as above (see also $[\mathrm{LR}])$. Namely, one fixes the element $B \in N\left(X^{\Delta}\right)$ corresponding to an isomorphism $X^{\nabla} \rightarrow X^{\Delta}$. Then $\psi_{B}(X)=\psi\left(\operatorname{Tr}_{X} \nabla(X B)\right)$ is a unitary character of $N\left(X^{\nabla}\right)$, and one has the corresponding generalized Jacquet integral $\tilde{\mathcal{W}}_{\psi, B}(\chi, s)$ on $I_{\tilde{P}\left(X^{\Delta}\right), \psi}(\chi, s)$. By a multiplicity 1 result, one has

$$
\tilde{\mathcal{W}}_{\psi, B}\left(\chi^{-1},-s\right) \circ \tilde{M}(\chi, s)=\tilde{c}_{\psi, B}(\chi, s) \cdot \tilde{\mathcal{W}}_{\psi, B}(\chi, s)
$$

for some meromorphic function $\tilde{c}_{\psi, B}(\chi, s)$. Then the normalized intertwining operator is defined by

$$
\tilde{M}_{\psi, B}^{*}(\chi, s)=\tilde{c}_{\psi, B}(\chi, s)^{-1} \cdot \tilde{M}(\chi, s) .
$$

Finally, one defines a standard $\gamma$-factor $\tilde{\gamma}(s, \tau \times \chi, \psi)$ by the local functional equation

$$
\begin{aligned}
\tilde{Z}_{X}\left(-s, \chi^{-1}, \tilde{M}_{\psi}^{*}(\chi, s)(\tilde{f}), v, v^{\vee}\right) \\
=\tilde{\gamma}(s+1 / 2, \tau \times \chi, \psi) \cdot(\tau \otimes \chi)\left(-1_{X} \Delta\right) \\
\quad \cdot \chi(\operatorname{det} B) \cdot|\operatorname{det} B|^{2 s} \tilde{Z}_{X}\left(s, \chi, \tilde{f}, v, v^{\vee}\right),
\end{aligned}
$$

where $\operatorname{det} B$ is defined in the same way as in the symplectic or metaplectic case. As before, one has the analogue of Corollary 4.2, which ensures that the $\gamma$-factor here is independent of the scaling of $B$. 


\subsection{Relation to linear case}

Now one has the linear version of the above construction, as detailed in [LR]. Thus, for the given representation $\tau$ of $\operatorname{GL}(X)$, one has the zeta integral $Z_{X}(s, \chi)\left(f, v^{\vee}, v\right)$, the normalized intertwining operator $M_{\psi, B}^{*}(\chi, s)$, and the $\gamma$-factor $\gamma_{X}(s, \tau \times \chi, \psi)$ defined by

$$
\begin{aligned}
Z_{X}\left(-s, \chi^{-1}\right)\left(M_{\psi, B}^{*}(\chi, s) f, v^{\vee}, v\right) & \\
= & \gamma_{X}(s+1 / 2, \tau \times \chi, \psi) \cdot(\tau \otimes \chi)\left(-1_{X^{\Delta}}\right) \\
& \quad \chi(\operatorname{det} B) \cdot|\operatorname{det} B|^{2 s} \cdot Z_{X}\left(s, \chi, f, v^{\vee}, v\right) .
\end{aligned}
$$

We note here a typo in [LR, (25)], where on the right-hand side of the above equation they had $\tau(-1)$ instead of $(\tau \otimes \chi)(-1)$. Moreover, it was shown in $[\mathrm{LR}]$ that

$$
\gamma_{X}(s, \tau \times \chi, \psi)=\gamma^{\mathrm{GJ}}(s, \tau \times \chi, \psi) \cdot \gamma^{\mathrm{GJ}}\left(s, \tau^{\vee} \times \chi, \psi\right),
$$

where $\gamma^{\text {GJ }}$ refers to the $\gamma$-factors of Godement-Jacquet. If one examines the proof of this identity in [LR], one sees that it is the definition using $(\tau \otimes \chi)(-1)$ which leads to this desired relation.

The goal of this section is to relate the nonlinear doubling zeta integral $\tilde{Z}_{X}$ and the $\gamma$-factor $\tilde{\gamma}_{X}(s, \tau \times \chi, \psi)$ defined in the previous section to the linear versions.

We first note that there is a natural $\tilde{G L}(\mathbb{X})$-equivariant isomorphism

$$
\iota(\chi, s): I_{P\left(X^{\Delta}\right)}(\chi, s) \otimes \chi_{\psi} \cong I_{\tilde{P}\left(X^{\Delta}\right), \psi}(\chi, s)
$$

given by

$$
f \mapsto \tilde{f}=f \cdot \chi_{\psi} .
$$

The following lemma is easy to check.

Lemma 7.1. One has

$$
\tilde{\mathcal{W}}_{\psi, B}(\chi, s) \circ \iota(\chi, s)=\mathcal{W}_{\psi, B}(\chi, s)
$$

and

$$
\tilde{M}(\chi, s) \circ \iota(\chi, s)=\chi_{\psi}\left(-1_{X}\right) \cdot \iota\left(\chi^{-1},-s\right) \circ M(\chi, s) .
$$

Thus,

$$
\tilde{c}_{\psi, B}(\chi, s)=\chi_{\psi}\left(-1_{X}\right) \cdot c_{\psi, B}(\chi, s),
$$


so that

$$
\tilde{M}_{\psi, B}^{*}(\chi, s) \circ \iota(\chi, s)=\iota\left(\chi^{-1},-s\right) \circ M_{\psi, B}^{*}(\chi, s) .
$$

Moreover,

$$
\tilde{Z}_{X}\left(s, \chi, \iota(\chi, s)(f), v, v^{\vee}\right)=2 \cdot Z_{X}\left(s, \chi, f, v, v^{\vee}\right) .
$$

The lemma immediately implies the following.

Proposition 7.2. One has

$$
\tilde{\gamma}_{X}(s, \tau \times \chi, \psi)=\gamma_{X}(s, \tau \times \chi, \psi) .
$$

In particular, $\tilde{\gamma}_{X}(s, \tau \times \chi, \psi)$ is independent of the choice of the isomorphism $B$ and not just independent of the scaling of $B$.

\section{§8. Main theorem}

Now we come to the main theorem of this paper.

THEOREM 8.1. One has the following.

(i) (Multiplicativity) Suppose that

$$
\sigma \subset \operatorname{Ind}_{\tilde{P}(X), \psi}^{\mathrm{Mp}(W)} \tau \otimes \sigma_{0},
$$

where $\tilde{P}(X)$ is a maximal parabolic subgroup with Levi factor $\mathrm{GL}(X) \times{ }_{\mu_{2}} \mathrm{Mp}\left(W_{0}\right), \tau$ is an irreducible representation of $\mathrm{GL}(X)$, and $\sigma_{0}$ is an irreducible genuine representation of $\operatorname{Mp}\left(W_{0}\right)$. Then

$$
\gamma_{W}(s, \sigma \times \chi, \psi)=\gamma_{X}(s, \tau \times \chi, \psi) \cdot \gamma_{W_{0}}\left(s, \sigma_{0} \times \chi, \psi\right) .
$$

(ii) (Variation of $\psi$ ) If $a \in F^{\times}$and $\psi_{a}(x)=\psi(a x)$, then

$$
\gamma_{W}\left(s, \sigma \times \chi, \psi_{a}\right)=\chi(a)^{2 n} \cdot|a|^{2 n(s-1 / 2)} \cdot \gamma_{W}\left(s, \sigma \times \chi_{a} \chi, \psi\right) .
$$

(iii) (Outer automorphism) Let $\sigma^{a}$ denote the twist of $\sigma$ by an outer automorphism of $\mathrm{Mp}(W)$ given by conjugation by an element in the similitude group $\operatorname{GSp}(W)$ of similitude a. Then one has

$$
\gamma_{W}\left(s, \sigma^{a} \times \chi, \psi\right)=\gamma_{W}\left(s, \sigma \times \chi_{a} \chi, \psi\right) .
$$

(iv) (Duality) One has

$$
\gamma_{W}\left(s, \sigma^{\vee} \times \chi, \psi\right)=\gamma_{W}\left(s, \sigma \times \chi, \psi_{-1}\right)=\gamma_{W}\left(s, \sigma \times \chi_{-1} \chi, \psi\right) .
$$


(v) (Functional equation) One has

$$
\gamma_{W}(s, \sigma \times \chi, \psi) \cdot \gamma_{W}\left(1-s, \sigma^{\vee} \times \chi^{-1}, \psi_{-1}\right)=1 .
$$

(vi) (Archimedean case) Suppose that $F$ is archimedean so that (by the analogue of the subrepresentation theorem) $\sigma$ is contained in a principal series representation $I_{\tilde{B}, \psi}\left(\chi_{1}, \ldots, \chi_{n}\right)$; then

$$
\gamma_{W}(s, \sigma \times \chi, \psi)=\prod_{i=1}^{n} \gamma\left(s, \chi_{i} \chi, \psi\right) \cdot \gamma\left(s, \chi_{i}^{-1} \chi, \psi\right),
$$

where the $\gamma$-factors on the right-hand side are those of Tate.

(vii) (Global property) Suppose that $k$ is a number field with ring of adèles $\mathbb{A}$ and that $W$ is a symplectic space over $k$. Let $\sigma$ be a cuspidal representation of $\operatorname{Mp}(W)_{\mathbb{A}}$, let $\chi$ be a Hecke character of $\mathbb{A}^{\times}$, and let $\psi=\bigotimes_{v} \psi_{v}$ be a nontrivial additive character of $k \backslash \mathbb{A}$. Let $S$ be a finite set of places outside of which all data involved are nonarchimedean and unramified. Then one has the global functional equation

$$
L^{S}(s, \sigma \times \chi, \psi)=\prod_{v \in S} \gamma_{W}\left(s, \sigma_{v} \times \chi_{v}, \psi_{v}\right) \cdot L^{S}\left(1-s, \sigma^{\vee} \times \chi^{-1}, \psi\right),
$$

where

$$
L^{S}(s, \sigma \times \chi, \psi)=\prod_{v \notin S} L\left(s, \sigma_{v} \times \chi_{v}, \psi_{v}\right)
$$

(when $\operatorname{Re}(s)$ is sufficiently large) is the partial L-function of $\sigma \times \chi$ with respect to $\psi$.

Moreover, the functions $\gamma_{W}(s, \sigma, \chi, \psi)$ are characterized by properties (i), (ii), and (vii).

\section{§9. Proof of the main theorem}

The rest of the paper is devoted to the proof of Theorem 8.1.

\subsection{Multiplicativity}

The proof of multiplicativity is by the same argument as that in [LR]. Namely, one may apply [LR, Proposition 2 and Lemma 9] and relate the doubling zeta integral for $\sigma$ on $\operatorname{Mp}(W)$ to the doubling zeta integral for $\sigma_{0}$ on $\operatorname{Mp}\left(W_{0}\right)$ and the doubling zeta integral for $\tau_{\psi}=\chi_{\psi} \cdot \tau$ on $\tilde{\mathrm{GL}}(X)$. In 
particular, by the analogue of [LR, Proposition 2], one has

$$
\begin{aligned}
Z_{W, \psi}(s, \chi)\left(f, \alpha^{\vee}, \alpha\right)= & \int_{\tilde{P}(X) \times \tilde{P}(X) \backslash \mathrm{Mp}(W) \times \mathrm{Mp}(W)} Z_{W_{0} \times X, \psi}(s, \chi) \\
& \times\left(\Psi(\chi, s)(f)(g), \alpha^{\vee}(g), \alpha(g)\right) d g .
\end{aligned}
$$

Here, we have set

$$
Z_{W_{0} \times X, \psi}(s, \chi)=Z_{W_{0}, \psi}(s, \chi) \otimes \tilde{Z}_{X, \psi}(s, \chi)
$$

and we have regarded $\alpha \in \sigma \subset \operatorname{Ind}_{\tilde{P}(X), \psi}^{\mathrm{Mp}(W)} \tau \otimes \sigma_{0}$ as a function on $\operatorname{Mp}(W)$ taking values in $\tau \otimes \sigma_{0}$. Moreover, $\Psi(\chi, s)$ is an intertwining operator

$$
\Psi(\chi, s): I_{\tilde{P}\left(W^{\Delta}\right), \psi}(\chi, s) \longrightarrow I_{\tilde{P}(\mathbb{X}), \psi}\left(I_{\tilde{P}\left(W_{0}^{\Delta}\right), \psi}(\chi, s) \otimes I_{\tilde{P}\left(X^{\Delta}\right), \psi}(\chi, s)\right)
$$

as defined in [LR].

Furthermore, in the normalization of the intertwining operator for $\operatorname{Mp}(\mathbb{W})$, let us choose the element $A \in \operatorname{Hom}\left(W^{\nabla}, W^{\Delta}\right)$ so that $A\left(X^{\nabla}\right)=$ $X^{\Delta}$, in which case we also have $A\left(W_{0}^{\nabla}\right) \subset W_{0}^{\Delta}$. Thus, the restriction of $A$ to $X^{\nabla}$ determines an element

$$
B \in \operatorname{Hom}\left(X^{\nabla}, X^{\Delta}\right)=N\left(X^{\Delta}\right) .
$$

Similarly, the restriction of $A$ to $W_{0}^{\nabla}$ determines an element

$$
A_{0} \in \operatorname{Hom}\left(W_{0}^{\nabla}, W_{0}^{\Delta}\right)=N\left(W_{0}^{\Delta}\right) .
$$

By [LR, Lemma 9], one has

$$
\Psi\left(\chi^{-1},-s\right) \circ M_{W, \psi, A}^{*}(\chi, s)=\operatorname{Ind}_{\tilde{P}(\mathbb{X})}^{\mathrm{Mp}(\mathbb{W})}\left(M_{W_{0} \times X, \psi, A_{0} \otimes B}^{*}(\chi, s)\right) \circ \Psi(\chi, s) .
$$

Hence, we have

$$
\begin{aligned}
Z_{W, \psi}(- & \left.s, \chi^{-1}\right)\left(M_{W, \psi, A}^{*}(\chi, s)(f), \alpha^{\vee}, \alpha\right) \\
= & \int_{\tilde{P}(X) \times \tilde{P}(X) \backslash \operatorname{Mp}(W) \times \operatorname{Mp}(W)} Z_{W_{0} \times X}(s, \chi) \\
& \times\left(M_{W_{0} \times X, \psi, A_{0} \otimes B}^{*}(\chi, s)(\Psi(\chi, s)(f)(g)), \alpha^{\vee}(g), \alpha(g)\right) d g .
\end{aligned}
$$

Now apply the local functional equations of the two zeta integrals in (9.2) and compare with (9.1). Noting that

$$
z_{\psi}(\sigma)=z_{\psi}\left(\sigma_{0}\right) \cdot \tau(-1)
$$


and that

$$
\operatorname{det} A=\operatorname{det} A_{0} \cdot \operatorname{det} B \cdot \operatorname{det}(-B)=(-1)^{\operatorname{dim} X} \operatorname{det}\left(A_{0}\right) \cdot(\operatorname{det} B)^{2},
$$

one deduces that

$$
\gamma_{W}(s, \sigma \times \chi, \psi)=\gamma_{W_{0}}\left(s, \sigma_{0} \times \chi, \psi\right) \cdot \tilde{\gamma}_{X}(s, \tau \times \chi, \psi) .
$$

The result then follows by Proposition 7.2.

\subsection{Archimedean case}

We note that statement (vi) of the main theorem follows immediately from statement (i), as a consequence of the subrepresentation theorem.

\subsection{Variation of $\psi$}

Now we are ready to prove statement (ii) of the main theorem. We note that

$$
\begin{aligned}
I_{\tilde{P}\left(W^{\Delta}\right), \psi_{a}}(\chi, s) & =I_{\tilde{P}\left(W^{\Delta}\right), \psi}\left(\chi \chi_{a}, s\right), \\
Z_{W, \psi_{a}}(s, \chi) & =Z_{W, \psi}\left(s, \chi \chi_{a}\right), \\
M_{\psi_{a}}(\chi, s) & =M_{\psi}\left(\chi \chi_{a}, s\right), \\
z_{\psi_{a}}(\sigma) & =z_{\psi}(\sigma) \cdot \chi_{a}(-1)^{n} .
\end{aligned}
$$

Thus,

$$
M_{\psi_{a}, A}^{*}(\chi, s)=\frac{c_{\psi, A}\left(s, \chi \chi_{a}\right)}{c_{\psi_{a}, A}(s, \chi)} \cdot M_{\psi, A}^{*}\left(\chi \chi_{a}, s\right) .
$$

On comparing the local functional equations for $Z_{W, \psi_{a}}(s, \chi)$ and $Z_{W, \psi}\left(s, \chi \chi_{a}\right)$, one concludes that

$$
\gamma_{W}\left(s+1 / 2, \sigma \times \chi, \psi_{a}\right) / \gamma_{W}\left(s+1 / 2, \sigma \times \chi_{a} \chi, \psi\right)=\frac{c_{\psi, A}\left(s, \chi \chi_{a}\right)}{c_{\psi_{a}, A}(s, \chi)} .
$$

Thus, (ii) holds (for any given $\sigma$ ) if and only if

$$
c_{\psi_{a}, A}(s, \chi)=|a|^{-2 n s} \cdot \chi(a)^{-2 n} \cdot c_{\psi, A}\left(s, \chi \chi_{a}\right) .
$$

This follows from the first and second identities in Proposition 4.1. However, we can prove this identity without resorting to Proposition 4.1 (which was taken as a black box from the unpublished work of Sweet [Sw]). Indeed, it follows from the above that if the identity in (ii) holds for one particular $\sigma$, then it holds for arbitrary $\sigma$. Now, by multiplicativity (i.e., (i)), it is easy to see that the identity in (ii) holds for principal series representations induced from the Borel subgroup $\tilde{B}$ of $\operatorname{Mp}(W)$. Hence, we are done with (ii). 


\subsection{Outer automorphism of $\mathrm{Mp}(\mathbb{W})$}

To address statement (iii) of the main theorem, we need to recall some facts about outer automorphisms of $\operatorname{Mp}(W)$. We follow the treatment of Szpruch $[\mathrm{Sz}]$. Consider the element $g_{a}$ of GSp(W) which acts as identity on $W^{\Delta}$ and by the scalar $a \in F^{\times}$on $W^{\nabla}$. Then $g_{a}$ has similitude $a$. Conjugation by $g_{a}$ defines an (outer) automorphism of $\mathrm{Sp}(\mathbb{W})$, which preserves the maximal parabolic $P\left(W^{\Delta}\right)$. It is a basic fact that this outer automorphism has a unique lift to $\mathrm{Mp}(\mathbb{W})$; we will denote this lifted automorphism of $\operatorname{Mp}(\mathbb{W})$ by $\nu_{a}$. Thus,

$$
\nu_{a}(h, \epsilon)=\left(g_{a} h g_{a}^{-1}, \epsilon_{a}(h) \cdot \epsilon\right)
$$

for some $\epsilon_{a}(h)= \pm 1$. The following lemma records some facts about the function $\epsilon_{a}$.

LEMMA 9.3. We have the following.

(i) If $h \in N\left(W^{\Delta}\right)$, then $\epsilon_{a}(h)=1$.

(ii) If $h \in M\left(W^{\Delta}\right)=\mathrm{GL}\left(W^{\Delta}\right)$, then $\epsilon_{a}(h)=\chi_{a}\left(\operatorname{det}_{W} \Delta h\right)$.

(iii) If $h=(1,-1) \in \operatorname{Sp}(W) \times \operatorname{Sp}(-W) \in \operatorname{Sp}(\mathbb{W})$, then $\epsilon_{a}(h)=\chi_{a}(-1)^{n}$.

It follows from the lemma that the automorphism $\nu_{a}$ induces a natural map

$$
\nu_{a}: I_{\tilde{P}\left(W^{\Delta}\right), \psi}(\chi, s) \longrightarrow I_{\tilde{P}\left(W^{\Delta}\right), \psi}\left(\chi_{a} \cdot \chi, s\right)
$$

given by

$$
\nu_{a}(f)(h)=f\left(\nu_{a}(h)\right)
$$

\subsection{Some computations}

Now we can compute the effect of the change of $\psi$ on various objects used in the definition of the $\gamma$-factors. Recall that

$$
I_{\tilde{P}\left(W^{\Delta}\right), \psi_{a}}(\chi, s)=I_{\tilde{P}\left(W^{\Delta}\right), \psi}\left(\chi \cdot \chi_{a}, s\right) .
$$

The following lemma relates the generalized Jacquet integrals $\mathcal{W}_{\psi_{a}, A}(\chi, s)$ and $\mathcal{W}_{\psi, A}(\chi, s)$.

Lemma 9.4. As linear functionals on $I_{\tilde{P}\left(W^{\Delta}\right), \psi_{a}}(\chi, s)$, one has

$$
\mathcal{W}_{\psi_{a}, A}(\chi, s)=|a|^{-n(2 n+1)} \cdot\left(\mathcal{W}_{\psi, A}(\chi, s) \circ \nu_{a^{-1}}\right)
$$


Proof. For $f \in I_{\tilde{P}\left(W^{\Delta}\right), \psi_{a}}(\chi, s)$, with $\operatorname{Re}(s) \gg 0$, one has

$$
\begin{aligned}
\mathcal{W}_{\psi_{a}, A}(\chi, s)(f) & =\int_{N\left(W^{\nabla}\right)} f(n) \cdot \psi_{a \cdot A}(n)^{-1} d n \\
& =\int_{N\left(W^{\nabla}\right)} f(n) \cdot \psi_{A}\left(\nu_{a}(n)\right)^{-1} d n \\
& =\int_{N\left(W^{\nabla}\right)} f\left(\nu_{a^{-1}}(n)\right) \cdot \psi_{A}(n)^{-1} \cdot|a|^{-n \cdot(2 n+1)} d n .
\end{aligned}
$$

The following lemma establishes the relation between $\nu_{a}$ and the standard intertwining operator $M(\chi, s)$.

Lemma 9.5. As operators from $I_{\tilde{P}\left(W^{\Delta}\right), \psi}(\chi, s)$ to $I_{\tilde{P}\left(W^{\Delta}\right), \psi}\left(\chi_{a} \chi^{-1},-s\right)$, one has

$$
\nu_{a} \circ M_{\psi}(\chi, s)=\chi_{-1}(a)^{n} \cdot \chi(a)^{2 n} \cdot|a|^{2 n s} \cdot M_{\psi_{a}}(\chi, s) \circ \nu_{a} .
$$

Proof. This is given by a similar computation as in the proof of Lemma 9.4:

$$
\begin{aligned}
\nu_{a}\left(M_{\psi}(\chi, s) f\right)(h) & =M(\chi, s)(f)\left(\nu_{a}(h)\right) \\
& =\int_{N\left(W^{\Delta}\right)} f\left(w n \cdot \nu_{a}(h)\right) d n \\
& =\int_{N\left(W^{\Delta}\right)} f\left(\nu_{a}\left(\nu_{a^{-1}}(w n) \cdot h\right)\right) d n
\end{aligned}
$$

Now we note that

$$
\nu_{a^{-1}}(w) \cdot w^{-1}=\left(a \cdot 1_{W^{\Delta}}, \chi_{a}(-1)^{n}\right) \in \tilde{\mathrm{GL}}\left(W^{\Delta}\right) \subset \mathrm{Mp}(\mathbb{W}) .
$$

Hence, we have

$$
\begin{aligned}
& \nu_{a}\left(M_{\psi}(\chi, s) f\right)(h) \\
& \quad=\int_{N\left(W^{\Delta}\right)} \chi_{-1}(a)^{n} \cdot \chi(a)^{2 n} \cdot|a|^{2 n s} \cdot f\left(\nu_{a}(w n h)\right) d n \\
& \quad=\chi_{-1}(a)^{n} \cdot \chi(a)^{2 n} \cdot|a|^{2 n s} \cdot M_{\psi_{a}}(\chi, s) \circ \nu_{a} .
\end{aligned}
$$

Corollary 9.6. One has

$$
c_{\psi_{a}, A}(\chi, s)=\chi_{-1}(a)^{n} \cdot \chi(a)^{-2 n} \cdot|a|^{-2 n s} \cdot c_{\psi, A}(\chi, s)
$$


and

$$
\nu_{a} \circ M_{\psi_{a}, A}^{*}(\chi, s)=M_{\psi, A}^{*}(\chi, s) \circ \nu_{a} .
$$

In particular, we have given an independent proof of the second identity in Proposition 4.1. Together with the results of Section 9.3, we have thus proved the first two identities of Proposition 4.1, independently of [Sw]. The third identity in Proposition 4.1 can be checked by computations analogous to those in Lemmas 9.4 and 9.5.

\subsection{Outer automorphism}

Let us write

$$
W=X \oplus X^{*},
$$

with $X$ maximal isotropic. Then one has an outer automorphism of $\operatorname{Sp}(W)$ given by conjugation by the element which acts as 1 on $X$ and $a$ on $X^{*}$. The lifting of this to $\operatorname{Mp}(W)$ is denoted by $\mu_{a}$. Moreover, the outer automorphism $\mu_{a} \times \mu_{a}$ on $\operatorname{Mp}(W) \times{ }_{\mu_{2}} \operatorname{Mp}(W)$ is the restriction of the outer automorphism of $\mathrm{Mp}(\mathbb{W})$ given by the conjugation of the element of $\operatorname{GSp}(\mathbb{W})$, which acts as 1 on $\mathbb{X}=X^{\Delta}+X^{\nabla}$ and as $a$ on $\mathbb{X}^{*}=\left(X^{*}\right)^{\Delta}+\left(X^{*}\right)^{\nabla}$. We denote this outer automorphism of $\mathrm{Mp}(\mathbb{W})$ by $\mu_{a}$ as well.

For any irreducible representation $\sigma$ of $\operatorname{Mp}(W)$, the twisted representation $\sigma^{a}$ is realized on the same space as $\sigma$, but the action of $\operatorname{Mp}(W)$ is defined by

$$
\sigma^{a}(g)=\sigma\left(\mu_{a}^{-1}(g)\right)
$$

Now consider the local zeta integral for $\sigma^{a}$ :

$$
\begin{aligned}
Z_{W, \psi}\left(s, \chi, \sigma^{a}\right)\left(f, v^{\vee}, v\right) & =\int_{\mathrm{Mp}(W)} f(g, 1) \cdot\left\langle\left(\sigma^{a}\right)^{\vee}(g) v^{\vee}, v\right\rangle d g \\
& =\int_{\mathrm{Mp}(W)} f(g, 1) \cdot\left\langle\sigma^{\vee}\left(\mu_{a}^{-1}(g)\right)\left(v^{\vee}\right), v\right\rangle d g \\
& =\int_{\mathrm{Mp}(W)} f\left(\mu_{a}(g, 1)\right) \cdot\left\langle\sigma^{\vee}(g)\left(v^{\vee}\right), v\right\rangle d g \\
& =\int_{\mathrm{Mp}(W)} f\left(\mu_{a} \nu_{a}^{-1} \nu_{a}(g, 1)\right) \cdot\left\langle\sigma^{\vee}(g)\left(v^{\vee}\right), v\right\rangle d g .
\end{aligned}
$$

Now it is easy to see that $\mu_{a} \nu_{a}^{-1}$ is the inner automorphism given by the element $m_{a}$ in the Levi subgroup $\operatorname{GL}\left(W^{\Delta}\right)=M\left(W^{\Delta}\right)$ of $P\left(W^{\Delta}\right)$ which acts 
as 1 on $X^{\Delta}$ and as $a$ on $\left(X^{*}\right)^{\Delta}$. Thus, we have

$$
\begin{aligned}
Z_{W, \psi}\left(s, \chi, \sigma^{a}\right)\left(f, v^{\vee}, v\right)= & \int_{\mathrm{Mp}(W)} f\left(m_{a} \nu_{a}(g, 1) m_{a}^{-1}\right) \cdot\left\langle\sigma^{\vee}(g)\left(v^{\vee}\right), v\right\rangle d g \\
= & \delta_{P\left(W^{\Delta}\right)}\left(m_{a}\right)^{1 / 2} \cdot \chi_{\psi}\left(m_{a}\right) \cdot|a|^{n s} \cdot \chi(a)^{n} \\
& \cdot Z_{W, \psi_{a}}(s, \chi, \sigma)\left(\nu_{a}\left(m_{a}^{-1} f\right), v^{\vee}, v\right) .
\end{aligned}
$$

We have thus shown the following.

LEMMA 9.7. One has

$$
\begin{aligned}
Z_{W, \psi}\left(s, \chi, \sigma^{a}\right)= & \delta_{P\left(W^{\Delta}\right)}\left(m_{a}\right)^{1 / 2} \cdot \chi_{\psi}\left(m_{a}\right) \cdot|a|^{n s} \\
& \cdot \chi(a)^{n} \cdot Z_{W, \psi_{a}}(s, \chi, \sigma) \circ\left(\nu_{a} \circ m_{a}^{-1} \otimes 1_{\sigma^{\vee}} \otimes 1_{\sigma}\right) .
\end{aligned}
$$

Similarly, one has

$$
\begin{aligned}
Z_{W, \psi}\left(-s, \chi^{-1}, \sigma^{a}\right) \circ M_{\psi, A}^{*}(s, \chi) \\
=\delta_{P\left(W^{\Delta}\right)}\left(m_{a}\right)^{1 / 2} \cdot \chi_{\psi}\left(m_{a}\right) \cdot|a|^{-n s} \cdot \chi(a)^{-n} \\
\quad \cdot Z_{W, \psi_{a}}\left(-s, \chi^{-1}, \sigma\right) \circ\left(\nu_{a} \circ m_{a}^{-1} \circ M_{\psi, A}^{*}(s, \chi)\right) \\
=\delta_{P\left(W^{\Delta}\right)}\left(m_{a}\right)^{1 / 2} \cdot \chi_{\psi}\left(m_{a}\right) \cdot|a|^{-n s} \cdot \chi(a)^{-n} \\
\quad \cdot Z_{W, \psi_{a}}\left(-s, \chi^{-1}, \sigma\right) \circ\left(M_{\psi_{a}, A}^{*}(s, \chi) \circ \nu_{a} \circ m_{a}^{-1}\right),
\end{aligned}
$$

where the last equality follows by Corollary 9.6.

By dividing this identity by that in Lemma 9.7 and noting that

$$
z_{\psi}\left(\sigma^{a}\right)=z_{\psi_{a}}(\sigma)
$$

we deduce that

$$
\gamma_{W}\left(s, \sigma^{a} \times \chi, \psi\right)=|a|^{-2 n s} \cdot \chi(a)^{-2 n} \cdot \gamma_{W}\left(s, \sigma \times \chi, \psi_{a}\right),
$$

which gives the identity in (iii) in view of (ii).

\subsection{Duality}

Statement (iv) of the main theorem follows from statement (iii), on noting that the contragredient of $\sigma$ is simply the twist of $\sigma$ by an outer automorphism associated to an element of $\operatorname{GSp}(W)$ of similitude -1 (see [MVW] and [Sun]). 


\subsection{Global property}

We now deal with the global functional equation, and we use the notation in statement (vi). For this, we need to introduce the global zeta integral associated to a cuspidal representation $\sigma=\bigotimes_{v} \sigma_{v}$ of $\operatorname{Mp}(W)_{\mathbb{A}}$. Consider the global degenerate principal series representation $I_{\tilde{P}\left(W^{\Delta}\right), \psi}(\chi, s)$ of $\mathrm{Mp}(\mathbb{W})_{\mathbb{A}}$ and its associated Eisenstein series map

$$
E_{\psi}(s, \chi): I_{\tilde{P}\left(W^{\Delta}\right), \psi}(\chi, s) \rightarrow \mathcal{A}(\mathrm{Mp}(\mathbb{W})),
$$

which is a meromorphic function of $s$. Here $\mathcal{A}(\mathrm{Mp}(\mathbb{W}))$ is the space of automorphic forms on $\operatorname{Mp}(W)_{\mathbb{A}}$, and the basic theory of Eisenstein series for nonlinear covering groups has been developed in [MW]. Then the global zeta integral

$$
Z_{W, \psi}(s, \chi): I_{\tilde{P}\left(W^{\Delta}\right), \psi}(\chi, s) \otimes \sigma^{\vee} \otimes \sigma \longrightarrow \mathbb{C}
$$

is defined by

$$
\begin{aligned}
Z_{W, \psi}(s, \chi)\left(f, \varphi^{\vee}, \varphi\right) & \\
= & \int_{\left(\operatorname{Mp}(W)_{k} \times \operatorname{Mp}(W)_{k}\right) \backslash\left(\operatorname{Mp}(W)_{\mathbb{A}} \times \operatorname{Mp}(W)_{\mathbb{A}}\right)} E_{\psi}(s, \chi)(f)\left(g_{1}, g_{2}\right) \\
& \cdot \varphi^{\vee}\left(g_{1}\right) \cdot \varphi\left(g_{2}\right) d g_{1} d g_{2} .
\end{aligned}
$$

The following proposition summarizes the key properties of this global zeta integral.

Proposition 9.8. We have the following.

(i) For $\operatorname{Re}(s)$ sufficiently large, one has a factorization

$$
Z_{W, \psi}(s, \chi)=\bigotimes_{v} Z_{W, \psi_{v}}\left(s, \chi_{v}\right)
$$

as linear functionals on $I_{\tilde{P}\left(W^{\Delta}\right), \psi}(\chi, s) \otimes \sigma^{\vee} \otimes \sigma$.

(ii) If $S$ is a sufficiently large finite set of places of $k$ outside of which all data are unramified and $\left(f, \varphi, \varphi^{\vee}\right)$ is the spherical vector, then one has the equality of meromorphic functions of $s$

$$
Z_{W, \psi}(s, \chi)\left(f, \varphi^{\vee}, \varphi\right)=\left(\prod_{v \in S} Z_{W, \psi_{v}}\left(s, \chi_{v}\right)\left(f_{v}, \varphi_{v}^{\vee}, \varphi_{v}\right)\right) \cdot \frac{L^{S}(s, \sigma \times \chi, \Psi)}{d^{S}(s, \chi)},
$$

where $d^{S}(s, \chi)$ is the product of partial Hecke L-functions as defined in Proposition 6.1. 
(iii) One has the global functional equation

$$
Z_{W, \psi}\left(-s, \chi^{-1}\right) \circ\left(M_{\psi}(s, \chi) \otimes 1_{\sigma} \otimes 1_{\sigma}\right)=Z_{W, \psi}(s, \chi),
$$

where

$$
M_{\psi}(s, \chi): I_{\tilde{P}\left(W^{\Delta}\right), \psi}(\chi, s) \longrightarrow I_{\tilde{P}\left(W^{\Delta}\right), \psi}\left(\chi^{-1},-s\right)
$$

is the standard global intertwining operator.

Proof. Statement (i) is due to Piatetski-Shapiro and Rallis [GPSR, part A]. Statement (ii) is a direct consequence of Proposition 6.1. Statement (iii) is a consequence of the functional equation of Eisenstein series (see [MW]).

In addition, we need to relate the global intertwining operator $M_{\psi}(s, \chi)$ with the normalized local intertwining operators $M_{\psi_{v}}^{*}\left(s, \chi_{v}\right)$. We fix a rational element $A \in N\left(W^{\Delta}\right)(k)$ and thus obtain the automorphic character $\psi_{A}$ of $N\left(W^{\nabla}\right)(\mathbb{A})$ defined as in the local case. We consider the associated Fourier coefficient map on $I_{\tilde{P}\left(W^{\Delta}\right), \psi}(\chi, s)$ defined by

$$
\mathcal{W}_{\psi}(s, \chi)(f)=\int_{N\left(W^{\nabla}\right)(k) \backslash N\left(W^{\nabla}\right)(\mathbb{A})} E_{\psi}(s, \chi)(f)(n) \cdot \psi_{A}(n)^{-1} d n .
$$

The following proposition summarizes the properties of $\mathcal{W}_{\psi_{A}}(s, \chi)$.

Proposition 9.9.

(i) One has

$$
\mathcal{W}_{\psi, A}(s, \chi)\left(M_{\psi}(s, \chi) f\right)=\mathcal{W}_{\psi, A}(s, \chi)(f) .
$$

(ii) When $\operatorname{Re}(s)$ is sufficiently large, one has

$$
\mathcal{W}_{\psi, A}(s, \chi)(f)=\prod_{v} \mathcal{W}_{\psi_{v}}\left(s, \chi_{v}\right)\left(f_{v}\right)
$$

Proof. Statement (i) is a consequence of the functional equation of Eisenstein series (see $[\mathrm{MW}]$ ). Statement (ii) follows by unfolding the Eisenstein series when $\operatorname{Re}(s)$ is sufficiently large.

Now for each place $v$ of $k$, the element $A$ allows one to define the normalized intertwining operator

$$
M_{\psi_{v}, A}^{*}\left(s, \chi_{v}\right)=c_{\psi_{v}, A}\left(s, \chi_{v}\right)^{-1} \cdot M_{\psi_{v}}\left(s, \chi_{v}\right)
$$


such that

$$
\mathcal{W}_{\psi_{v}, A}\left(-s, \chi_{v}^{-1}\right) \circ M_{\psi_{v}, A}^{*}\left(s, \chi_{v}\right)=\mathcal{W}_{\psi_{v}, A}\left(s, \chi_{v}\right) .
$$

Morally speaking, the above proposition says that

$$
" \prod_{v} " c_{\psi_{v}, A}\left(s, \chi_{v}\right)=1
$$

so that

$$
M_{\psi}(s, \chi)=" \prod_{v} " M_{\psi_{v}, A}^{*}\left(s, \chi_{v}\right) .
$$

However, the Euler products above do not converge anywhere and have to be suitably interpreted. This is given by the following proposition, after taking into account the results of Proposition 6.1.

Corollary 9.10. For a sufficiently large finite set $S$ of places of $k$, one has

$$
\prod_{v \in S} c_{\psi_{v}, A}\left(s, \chi_{v}\right)=\prod_{j=1}^{n} \frac{L^{S}\left(2 j-2 s, \chi^{-2}\right)}{L^{S}\left(2 s-2 j+1, \chi^{2}\right)} .
$$

Proof. Consider the identity

$$
\mathcal{W}_{\psi, A}\left(-s, \chi^{-1}\right)\left(M_{\psi}(s, \chi) f\right)=\mathcal{W}_{\psi, A}(s, \chi)(f),
$$

with $f=\bigotimes_{v} f_{v}$ such that for all $v \notin S$, the conditions of Proposition 6.1 hold. Then Proposition 6.1 implies that

$$
\begin{aligned}
& \left(\prod_{v \in S} \mathcal{W}_{\psi_{v}, A}\left(-s, \chi_{v}^{-1}\right)\left(M\left(s, \chi_{v}\right)\left(f_{v}\right)\right)\right) \cdot \frac{\prod_{j=1}^{n} L^{S}\left(2 s-2 j+1, \chi^{2}\right)}{d^{S}(s, \chi) \cdot d^{S}\left(-s, \chi^{-1}\right)} \\
& \quad=\left(\prod_{v \in S} \mathcal{W}_{\psi_{v}, A}\left(s, \chi_{v}\right)\left(f_{v}\right)\right) \cdot \frac{1}{d^{S}(s, \chi)} .
\end{aligned}
$$

Thus, we have

$$
\begin{aligned}
& \left(\prod_{v \in S} c_{\psi_{v}, A}\left(s, \chi_{v}\right) \cdot \mathcal{W}_{\psi_{v}, A}\left(s, \chi_{v}\right)\left(f_{v}\right)\right) \cdot \frac{\prod_{j=1}^{n} L^{S}\left(2 s-2 j+1, \chi^{2}\right)}{d^{S}\left(-s, \chi^{-1}\right)} \\
& \quad=\prod_{v \in S} \mathcal{W}_{\psi_{v}, A}\left(s, \chi_{v}\right)\left(f_{v}\right) .
\end{aligned}
$$

Hence,

$$
\prod_{v \in S} c_{\psi_{v}, A}\left(s, \chi_{v}\right)=\prod_{j=1}^{n} \frac{L^{S}\left(2 j-2 s, \chi^{-2}\right)}{L^{S}\left(2 s-2 j+1, \chi^{2}\right)}
$$


Combining this with the global functional equation in Proposition 9.8(iii), the results of the above corollary and Proposition 6.1, and the local functional equation of the local zeta integral in Section 5, one deduces the desired result as in $[\mathrm{LR}]$.

\subsection{Uniqueness}

The uniqueness of the $\gamma$-factors considered in the main theorem is proved in the same way as in $[\mathrm{LR}]$.

Acknowledgments. The author first met Professor Hiroshi Saito in January 2003 when he visited the University of Kyoto for a conference. Professor Saito's warm hospitality will be fondly remembered. This paper was written during the author's visit to the National University of Singapore (NUS) in the fall of 2010. The author thanks NUS for providing an excellent working environment. Thanks are also due to Erez Lapid for several helpful e-mail exchanges.

\section{REFERENCES}

[GS] W. T. Gan and G. Savin, Representations of metaplectic groups I: epsilon dichotomy and local Langlands correspondence, to appear in Compos. Math., available at http://www.math.nus.edu.sg/ matgw/metaplectic-1.pdf (accessed 15 August 2012).

[GPSR] S. Gelbart, I. Piatetski-Shapiro, and S. Rallis, Explicit Constructions of Automorphic L-Functions, Lecture Notes in Math. 1254, Springer, Berlin, 1987.

[LR] E. Lapid and S. Rallis, "On the local factors of representations of classical groups" in Automorphic Representations, L-Functions and Applications: Progress and Prospects, Ohio State Univ. Math. Res. Inst. Publ. 11, de Gruyter, Berlin, 2005, 309-359.

[Li] J.-S. Li, Nonvanishing theorems for the cohomology of certain arithmetic quotients, J. Reine Angew. Math. 428 (1992), 177-217.

[MVW] C. Moeglin, M.-F. Vigneras, and J.-L. Waldspurger, Correspondances de Howe sur un corps p-adique, Lecture Notes in Math. 1291, Springer, Berlin, 1987.

[MW] C. Moeglin and J.-L. Waldspurger, Spectral Decomposition and Eisenstein Series, Cambridge Tracts in Math. 113, Cambridge University Press, Cambridge, 1995.

[PSR] I. Piatetski-Shapiro and S. Rallis, $\epsilon$ factor of representations of classical groups, Proc. Natl. Acad. Sci. USA 83 (1986), 4589-4593.

[R] R. Rao, On some explicit formulas in the theory of the Weil representation, Pacific J. Math. 157 (1993), 335-371.

[Sun] B. Y. Sun, Dual pairs and contragredients of irreducible representations, Pacific J. Math. 249 (2011), 485-494.

[Sw] J. Sweet, Functional equations of p-adic zeta integrals and representations of the metaplectic group, preprint, 1995.

[Sz] D. Szpruch, The Langlands-Shahidi method for the metaplectic group and applications, preprint, arXiv:1004.3516v1 [math.NT] 
[Y] S. Yamana, L-functions and theta correspondences for classical groups, preprint, 2011.

[Z] C. Zorn, Theta dichotomy and doubling epsilon factors for $\widetilde{\operatorname{SP}_{n}}(F)$, Amer. J. Math. 133 (2011), 1313-1364.

Department of Mathematics

National University of Singapore

Singapore 119076

matgwt@nus.edu.sg 